\title{
How to Identify Retinoblastoma in Pediatric Patients
}

\author{
Supalert Prakhunhungsit ${ }^{1,2}$ and Audina M Berrocal ${ }^{2}$ \\ 1. Retina Unit, Ophthalmology Department, Faculty of Medicine, Siriraj Hospital, Mahidol University, Bangkok, Thailand; \\ 2. Retina Department, Bascom Palmer Eye Institute, FL, US
}

DOI: https://doi.org/10.17925/USOR.2018.11.2.77

\section{Keywords}

Retinoblastoma identification, pediatric patients, ocular imaging

Disclosure: Supalert Prakhunhungsit and
Audina M Berrocal have nothing to
disclose in relation to this article.

Review Process: This article is a short opinion piece and has not been submitted to external peer reviewers but was reviewed by the editorial board for accuracy before publication.

Authorship: All named authors meet the International Committee of Medical Journal Editors (ICMJE) criteria for authorship of this manuscript, take responsibility for the integrity of the work as a whole, and have given final approval to the version to be published. open Access: This article is published under the Creative commons Attribution Noncommercial License, which permits any noncommercial use, distribution, adaptation, and reproduction provided the original author(s) and source are given appropriate credit. () The Author 2018.

Received: June 5, 2018

Published Online: October 15, 2018

Citation: US Ophthalmic Review. 2018;11(2):77-8

Corresponding Author: Audina M Berrocal, Retina Department, Bascom Palmer Eye Institute, 900 NW 17th street, Miami, FL 33136, US. E: aberrocal@med.miami.edu

Support: No funding was received in the publication of this article.
One of the most important differential diagnoses in the pediatric retina population is retinoblastoma. A detailed history, good eye examination, good ultrasonography, and any other ancillary tests are essential for making the correct diagnosis.

Retinoblastoma is the most common primary ocular tumor in pediatric patients. The incidence accounts for 1 in 20,000 live births and approximately 300 new cases per year in the US. ${ }^{1}$ Two-thirds of cases were diagnosed before the age of 2 years and it is rare for the diagnosis to present after 5 years of age. ${ }^{1}$ The improvement of treatment strategies evolving from enucleation or external beam radiation to more advanced globe-saving therapies in recent years has increased the survival rate of children with retinoblastoma to more than $95 \%$ in developed countries. ${ }^{2}$

The clinical pearls to identify the pediatric patients with retinoblastoma should start from a detailed medical history and a complete physical examination. The family history of retinoblastoma or other malignancies should be scrutinized, not only for an accurate diagnosis, but also to advise families. Genetic testing and genetic counseling is of utmost importance. A careful ocular examination, usually under general anesthesia, should be conducted to assess the intraocular findings and ascertain which layer of the retina is involved: preretinal, intraretinal, or subretinal layers. This will help in understanding what kind of tumor presentation we are dealing with.

\section{B-scan ultrasonography}

A B-scan ultrasonography of the tumor offers important information, but it is not always perfect. The heterogeneity and calcification detection within the intraocular mass from ultrasonography provide strong evidence for the diagnosis of retinoblastoma. Specifically, calcium within an intraocular mass in children before the age of 3 years is highly suspicious for retinoblastoma until proven otherwise. Although histopathological examination of enucleated globes showed approximately $95 \%$ of calcium deposit within the tumor, ${ }^{3}$ the ocular ultrasonography is not as sensitive as other advanced imaging modalities to detect the calcification. ${ }^{4}$ Echography can also detect subretinal fluid or seeding, tumor growth patterns, and in some circumstances, vitreous seeding. ${ }^{5}$

\section{Computed tomography scan and magnetic resonance imaging}

Most of the time, other imaging modalities such as computed tomography (CT) scan or magnetic resonance imaging (MRI), are needed. Moreover, CT scans and MRI can detect the extraocular extension and intracranial lesions in patients with trilateral disease, which leads to accurate disease staging and appropriate treatment thereafter. CT scans are one of the most effective methods of detecting calcification, but with the concern of radiation exposure in patients with retinoblastoma with germline mutation, it is generally avoided. ${ }^{6}$ The benefit of MRI over CT is the detection of the optic nerve invasion which is one of the prognostic factors in retinoblastoma. Furthermore, $\mathrm{MRI}$ is also superior to CT in terms of soft tissue delineation to uncover pineal tumors in trilateral 
retinoblastoma cases or, rarely, in the suprasellar region or leptomeningeal spread. ${ }^{7}$ However, MRI is less sensitive to detect intralesional calcification than $\mathrm{CT}$

\section{Wide-field fundus photography with intravenous fluorescein angiography}

Wide-field fundus photography with intravenous fluorescein angiography (IVFA) in the operating room is helpful in making a diagnosis, especially in cases with unusual presentation. Advanced endophytic or exophytic retinoblastoma is often associated with large vessel tortuosity and dilatation with small vessel telangiectasia and microaneurysm, intrinsic tumor vasculature and late leakage in IVFA photographs. ${ }^{8}$ On the other hand in the diffuse retinoblastoma it becomes more challenging to make the diagnosis. In these cases, IVFA demonstrates complex branching with tortuosity and telangiectatic vessels within the area of the mass. The feeder vessels show early termination and are variably visualized as a consequence of the overlying tumor. ${ }^{9}$ Furthermore, IVFA is also useful in differentiating retinoblastoma from Coats' disease, a common confounding entity. Retinoblastoma does not present telangiectatic vasculature with aneurysmal dilatation as it would in patients with Coats disease. IVFA is also capable of distinguishing retinoblastoma cases with Coats'-like response from Coats' disease. The anterior segment fluorescein angiograms, derived from IVFA, capturing the iris vasculature detail, is also worthwhile in guiding the diagnosis of advanced retinoblastoma. Iris neovascularization, present in advanced disease, will be enhanced by injected dye and consequently the extension of the disease in group $\mathrm{E}$ retinoblastoma can be detected. Additionally, the IVFA in the fellow eye also provides useful information for making a complete diagnosis in peculiar cases. The area of peripheral retinal avascularity in the other eye could point to familial exudative vitreoretinopathy (FEVR). In pediatric patients with a history of prematurity and vitreous hemorrhage, the IVFA findings in the other eye aid in making the correct diagnosis. Pruning of the vasculature with budding into the area of retinal nonperfusion could lead to the diagnosis of retinopathy of prematurity with FEVR. ${ }^{10}$ Recognition of atypical characteristics, i.e., older age, unilateral cases, and atypical color, is always advantageous to the critical use of wide-field photography with IVFA and diagnostic echography.

Once retinoblastoma is suspected, intravitreal injection and any intraocular surgeries should be strictly avoided as they can cause orbital extension. ${ }^{11}$ When intraocular surgery is performed in cases with atypical presentation, the aqueous, vitreous, or any removed tissue should be sent for histopathological examination. When in doubt of the diagnosis of retinoblastoma, a referral and consultation for a second opinion from a pediatric ocular oncologist is definitely a reasonable and recommended option.

\section{Conclusion}

As a pediatric retina specialist, the best advice in the diagnosis of atypical retinoblastoma is to THINK about it. If it is unusual, if it doesn't make sense to you STOP. Do more testing and if you cannot be sure then send the case to a pediatric ocular oncologist. Additionally, always look carefully at the other eye. $\square$
1. Ries LA, Smith MA, Gurney JG, et al. Cancer incidence and survival among children and adolescents: United States SEER Program 1975-1995. NIH Pub. No. 99-4649. Bethesda, MD, 1999.

2. Abramson DH. Retinoblastoma: saving life with vision. Annu Rev Med. 2014;65:171-84.

3. Froula PD, Bartley GB, Garrity JA, Forbes G. The differential diagnosis of orbital calcification as detected on computed tomographic scans. Mayo Clinic Proceedings. 1993:68:256-61.

4. Gallie BL, Zhao J, Vandezande K, et al. Global issues and opportunities for optimized retinoblastoma care. Pediatr Blood Cancer. 2007;49(S7):1083-90
5. Francis JH Marr BP Abramson DH Classification of vitreous seeds in retinoblastoma: correlations with patient, tumor, and treatment characteristics. Ophthalmology. 2016;123:1601-5.

6. Smith EV, Gragoudas ES, Kolodny NH, D'Amico DJ. Magnetic resonance imaging: an emerging technique for the diagnosis of ocular disorders. Int Ophthalmol. 1990;14:119-24.

7. de Graaf P, Göricke S, Rodjan F, et al. European Retinoblastoma Imaging Collaboration. Guidelines for imaging retinoblastoma: imaging principles and MRI standardization. Pediatr Radiol. 2012;42:2-14.

8. Kim JW, Ngai LK, Sadda S, et al. Retcam fluorescein angiography findings in eyes with advanced retinoblastoma. $\mathrm{Br} J$ Ophthalmol. 2014;98:1666-71.

9. Fernandez MP, Al-Holou SN, Fischer O, et al. Fluorescein angiography findings in diffuse retinoblastoma: two case reports with clinicopathologic correlation. J AAPOS. 2017;21:337-9.

10. John VJ, McClintic JI, Hess DJ, Berrocal AM. Retinopathy of prematurity versus familial exudative vitreoretinopathy: report on clinical and angiographic findings. Ophthalmic Surg Lasers Imaging Retina. 2016:47:14-9.

11. Ali MJ, Honavar SG, Reddy VA. Orbital retinoblastoma: Present status and future challenges-A review. Saudi J Ophthalmol. 2011;25:159-67. 